\title{
Cardiac conduction abnormalities preceding transoesophageal echocardiographic evidence of perivalvar extension of infection in a case of salmonella prosthetic valve endocarditis
}

\author{
Michael I Miyamoto, Adolph M Hutter Jr, Jonathan H Blum, David F Torchiana
}

\begin{abstract}
A 59 year old African-American man developed complete heart block in association with Salmonella enteritidis prosthetic valve endocarditis. Severe cardiac conduction abnormalities signalled the presence of perivalvar extension of infection before development of evidence of abscess by transoesophageal echocardiography. Cardiac conduction temporarily returned after debridement and aortic homograft placement. This case emphasises the value of electrocardiographic monitoring in the detection of perivalvar extension of infection complicating infective endocarditis, even in the era of sophisticated imaging modalities. (Heart 1997;78:416-418)
\end{abstract}

Keywords: endocarditis; Salmonella enteritidis; heart block

Disorders of cardiac conduction are known complications of infective endocarditis, occurring in approximately $10 \%$ of episodes of native valve endocarditis. ${ }^{1}$ Infections of various components of cardiac valves have been correlated with specific conduction disturbances, which are usually due to extension of infection into surrounding tissues, associated oedema, or bacterial myocarditis encroaching upon components of the cardiac conduction system. ${ }^{23}$ Unstable conduction abnormalities in native valve endocarditis have been shown to signal the presence of myocardial abscesses and therefore poorer outcome and greater need for surgical therapy. ${ }^{4}$

We report a case of Salmonella enteritidis prosthetic valve endocarditis, which is remarkable for the appearance of severe disturbances of cardiac conduction before the development of any marked anatomical abnormality by transoesophageal echocardiography (TOE), and the early recovery of cardiac conduction despite severe local tissue destruction by infection and extensive local surgical manipulation.

\section{Case report}

A 59 year old African-American man was admitted with presyncope preceded by abdominal pain, chills, and diarrhoea. He had a history of polymyositis for which he was maintained on prednisone and oral methotrexate. $\mathrm{He}$ had undergone aortic valve replacement with a $23 \mathrm{~mm}$ Carpentier-Edwards (Baxter Healthcare Corp, Deerfield, Illinois, USA) pericardial bioprosthesis 28 months before admission for mixed aortic stenosis and aortic insufficiency. The patient had been hospitalised five months previously for atypical chest pain, during which time he developed severe diarrhoea. Stool cultures grew abundant salmonella group D. He was treated with oral ciprofloxacin.

On the morning of the later admission, he developed lightheadedness, abdominal discomfort, and diarrhoea. Emergency medical personnel found him to be responsive with a systolic blood pressure of $70 \mathrm{~mm} \mathrm{Hg}$. In the emergency room, he was feverish $\left(39.6^{\circ} \mathrm{C}\right)$ with rigors. His heart rate was 91 beats/min. Lung fields were clear to auscultation. Jugular venous pressure was $8 \mathrm{~cm}$. There was a harsh grade II-VI systolic ejection murmur at the left upper sternal border radiating weakly to the carotids. There were no diastolic murmurs or extra heart sounds. Chest radiographs demonstrated clear lungs. Electrocardiogram demonstrated sinus tachycardia with 4:3 Wenckebach block, left atrial enlargement, and left ventricular hypertrophy. There was new right bundle branch block and old left anterior fascicular block. TOE demonstrated a well seated bioprosthesis in the aortic position with trace aortic insufficiency but no paravalvar leak (fig 1, top). There was a small echodensity associated with the left coronary cusp, suspicious for a small mobile vegetation. There was no evidence of ring abscess, and the remaining valves were unremarkable. There was concentric left ventricular hypertrophy. Almost immediately upon completion of this study, the patient's second degree atrioventricular block progressed to third degree block with
Accepted for publication 10 June 1997 


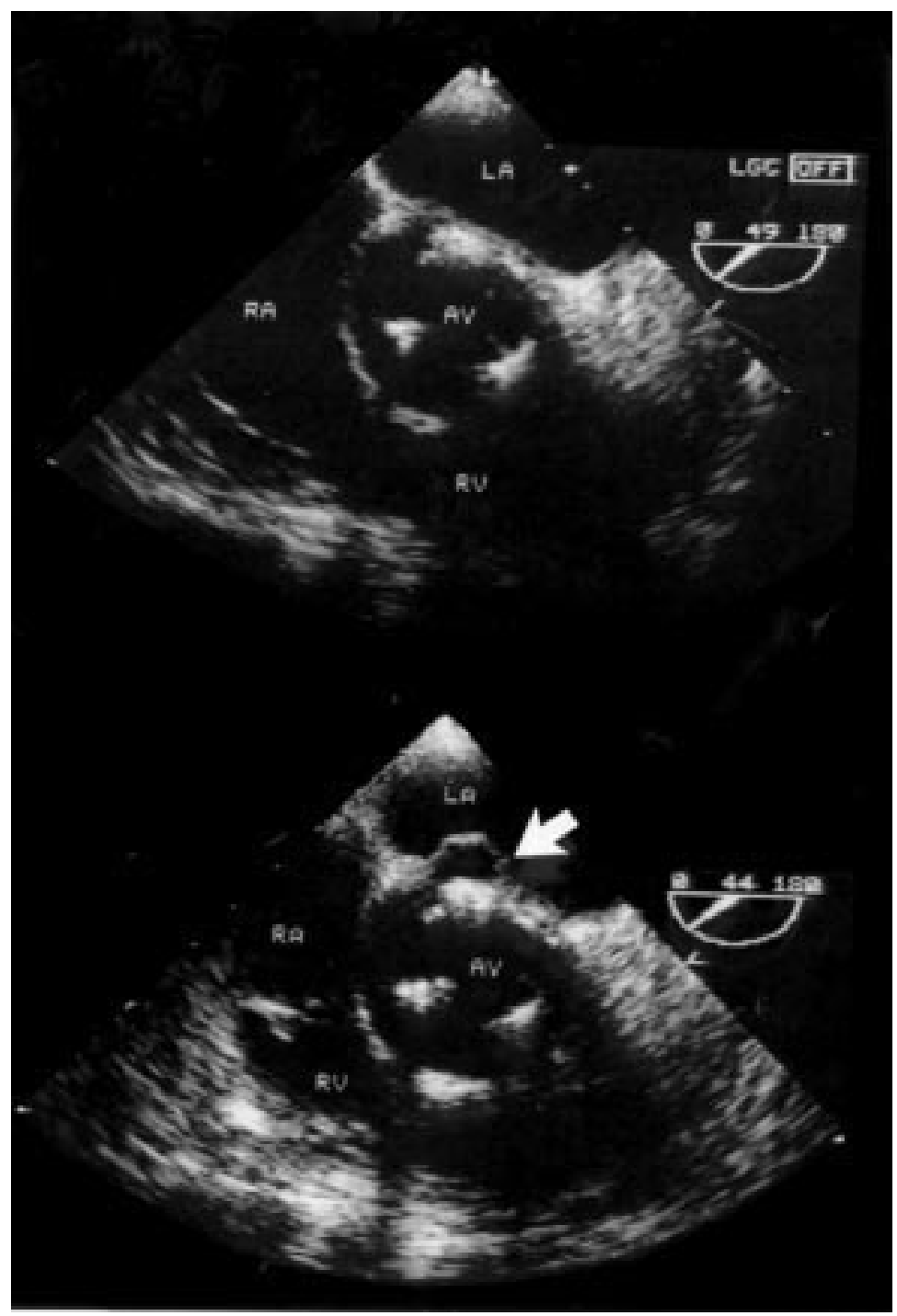

Figure 1 Transoesophageal echocardiograms immediately before the development of complete heart block (top) and four days later (bottom). In these short axis views above the level of the sewing ring of the pericardial bioprosthesis, an abscess cavity, not seen in the initial study, is clearly seen in the follow up study (arrow). AV, aortic valve; LA, left atrium; $R A$, right atrium; $R V$, right ventricle. left atrium was closed with a patch. A cryopreserved aortic homograft was placed, with reimplantation of the coronary arteries. Postoperatively, he remained in complete heart block, and arrangements were made to implant a permanent pacemaker. However, on postoperative day 4 , sinus rhythm with first degree atrioventricular block and left bundle branch block were noted. Epicardial pacing wires were removed, and the patient's cardiac conduction remained stable. He was discharged on postoperative day 8 on a six week course of intravenous ceftriaxone and an indefinite course of oral ofloxacin.

At a six month follow up visit the patient complained of dyspnoea on exertion. His physical examination was notable for a pulse rate of 46 beats/min. Electrocardiogram demonstrated second degree atrioventricular block with $2: 1$ conduction. He was hospitalised, and multiple blood cultures were obtained, all of which were negative. TOE demonstrated only postsurgical changes, mild central aortic insufficiency, and moderate mitral regurgitation with preserved left ventricular function. No vegetations or abscesses were seen. During treadmill exercise, 3:1 atrioventricular block developed, accompanied by marked dyspnoea. A rate responsive dual chamber permanent pacemaker was implanted. The patient reported his dyspnea on exertion to be greatly improved at three month follow up.

\section{Discussion}

Perivalvar extension of infection occurs more commonly with involvement of the aortic valve. $^{3}$ The presence of a valvar prosthesis further increases this risk, as the annulus is usually the primary site of infection. ${ }^{3}$ The incidence of perivalvar extension of infection with aortic prosthetic valve endocarditis in necropsy and surgical series varies widely from $4-100 \%$. $^{3}$ Consistent with the observation that cardiac conduction abnormalities correlate with perivalvar extension of infection, DiNubile, et al described a series of 211 episodes of native valve endocarditis in which 20 developed unstable conduction abnormalities. ${ }^{1}$ Pathological information was available for 12 of these cases, eight of which had extension of infection beyond the valve leaflets. ${ }^{1}$ However, only eight of 29 patients with pathologically confirmed extension of infection had conduction abnormalities. Thus, the presence of conduction abnormalities on the ECG is relatively specific but insensitive for perivalvar extension of infection. ${ }^{3}$

Given the proximity of the aortic valve to components of the cardiac conduction system, extension of infection from specific valve leaflets may give rise to predictable conduction system abnormalities. ${ }^{2}$ From electrocardiographic considerations alone, this patient was suspected to have extension of infection from the aortic valve into the ventricular septum. This was probably the case, even though TOE initially failed to suggest any abnormality in the tissues surrounding the aortic prosthesis.

TOE has been demonstrated to be quite sensitive for the detection of myocardial 
abscesses associated with native valve endocarditis. ${ }^{5}$ Acoustic shadowing can make detection of abnormalities more difficult in the presence of a valvar prosthesis. It is unknown whether patients with severe conduction system abnormalities and abscess are more likely to have abnormalities by TOE.

In the present case, electrocardiographic manifestations occurred very early in the course of extension of infection. Thus, this case illustrates the importance of conduction system abnormalities, when present, and particularly so with a known aggressive pathogen such as salmonella. Although imaging techniques are increasingly relied upon to guide management of endocarditis, the usefulness of electrocardiographic monitoring should not be forgotten.

Cardiac conduction initially recovered after debridement of the infected area and homograft placement. In our experience, conduction abnormalities of this severity associated with perivalvar extension of infection are virtually always irreversible, necessitating permanent pacemaker therapy. That the defect in atrioventricular conduction was initially reversible sug- gests a possible role for infection associated oedema, myocarditis or both, which resolved after the main focus of infection was removed. DiNubile suggested a duration of one week of persistent heart block as evidence of irreversibility, in the absence of drug effects or myocardial infarction. ${ }^{4}$ As this case illustrates, there are rare exceptions to this observation. Interestingly, although this patient's atrioventricular conduction temporarily recovered, the left bundle branch block persisted, and second degree heart block recurred six months later.

1 DiNubile MJ, Calderwood SB, Steinhaus DM, Karchmer AW. Cardiac conduction abnormalities complicating native
valve active infective endocarditis. Am 7 Cardiol valve active infect
$1986 ; 58: 1213-17$.

2 Hutter AM, Moellering RC. Assessment of the patient with suspected endocarditis. FAMA 1976;235:1603-6.

3 Carpenter JL. Perivalvular extension of infection in patients with infectious endocarditis. Rev Infect Dis 1991;13:12738 .

4 DiNubile MJ. Heart block during bacterial endocarditis: a review of the literature and guidelines for surgical intervention. Am f Med Sci 1984;287:30-2.

5 Daniel WG, Mugge A, Martin RP, Lindert O, Hausmann D, Nonnast-Daniel B, et al. Improvement in the diagnosis of Nonnast-Daniel $\mathrm{B}$, et al. Improvement in the diagnosis of
abscesses associated with endocarditis by transesophageal echocardiography. N Engl f Med 1991;324:795-800. 